Article

\title{
Vehicle Door Opening Control Model Based on a Fuzzy Inference System to Prevent Motorcycle-Vehicle Door Crashes
}

\author{
Cheng-Yong Huang (iD \\ Department of Arts and Design, National Dong Hwa University, Hualien 974301, Taiwan; \\ yong@gms.ndhu.edu.tw
}

check for updates

Citation: Huang, C.-Y. Vehicle Door Opening Control Model Based on a Fuzzy Inference System to Prevent Motorcycle-Vehicle Door Crashes. Sustainability 2021, 13, 12558. https:/ / doi.org/10.3390/su132212558

Academic Editors: Junfang Tian, Zhongxiang Feng and Shuxian $\mathrm{Xu}$

Received: 21 October 2021

Accepted: 11 November 2021

Published: 13 November 2021

Publisher's Note: MDPI stays neutral with regard to jurisdictional claims in published maps and institutional affiliations.

Copyright: (C) 2021 by the author. Licensee MDPI, Basel, Switzerland. This article is an open access article distributed under the terms and conditions of the Creative Commons Attribution (CC BY) license (https:// creativecommons.org/licenses/by/ $4.0 /)$.

\begin{abstract}
The goal of this research is to develop a fuzzy logic-based vehicle door control system to avoid motorcycle-vehicle door crash accidents. Accidents of this nature usually occur when the driver has parked the car, opens the door getting out of the car and collides with a motorcycle approaching from the rear, causing injury to the motorcyclist. In order to prevent such accidents, the fuzzy logic control system inputs the speed (MS) and safety distance (SD) of the motorcycle approaching from the rear, and then the fuzzy inference unit (FIU) calculates the clear output (Crisp) defuzzification Vehicle Door Opening Model (VDOM) value for the central locking system of the car, which can be used to trigger three modes, namely Danger Mode, Caution Mode and Warning Mode. In this study, the VDOM system is designed to trigger reasonable, reliable and consistent door control under different speeds of motorcycles coming from the rear and will be effectively applied to the door control of semi-automatic cars in the future.
\end{abstract}

Keywords: door crash; fuzzy logic control; central locking; injury prevention; mandatory Dutch Reach

\section{Introduction}

Factors including different modes of transportation and transportation facilities have made motorcycle-vehicle door crash accidents unique to Taiwan and have contributed to the high frequency of said traffic accidents on the island [1]. Taiwan has the highest motorcycle to car ratio, with the number of motorcycles being twice that of the number of cars [2]. Cars are also usually allowed to park on both sides of the road. When a car driver recklessly opens the door and hits an approaching motorcycle, this door crash is regarded as a motorcycle-vehicle right of way (ROW) collision [3]. Door collisions are usually caused by the driver's failure to notice the approaching motorcycle before opening the door, striking down the motorcyclist and causing injuries, and even exposing the victim to the risks of being run over by a vehicle coming from the rear, causing fatalities [4].

The ever-progressing collision avoidance system of cars is related to the development of detection techniques. It is a safety technology that utilises communication, control and information technology to detect the dynamic conditions around the vehicle to assist the car driver. As early as 2009, the American National Highway Traffic Safety Administration began studying the specifications of collision prevention warnings and lane deviation warnings [5], for instance, the active brake assist system and forward collision warning can also effectively reduce the incidence of rearward collisions [6]. As for the blind spot of the rear-view mirror, numerous car manufacturers including Volvo have installed a Blind Spot Detection system since 2003, which actively scans blind spots through the left- and right-side sensory radars to detect cars in the blind spots [7].

Regarding the door locks, cars with a central locking security system provide users the convenience of locking and unlocking doors via a simple push of buttons from a remote control. Car manufacturers mainly adopt two central locking systems, the automatic central locking system and the manual central locking system, to ensure smoother and safer door control operations [8]. Cars equipped with central locking will have all the doors 
automatically locked when the vehicle reaches the speed of $20 \mathrm{~km} / \mathrm{h}$ and unlocking when the driver shifts the gear lever to $P$ position. This prevents passengers from accidentally opening the door inside a moving vehicle. The current research on door control focuses predominantly on the prevention of thieves [8,9]. In terms of controlling the door switch to prevent passengers from suffering injuries in traffic accidents, Mathavan et al. proposed car fire detection to control the door opening hardware application, through temperature and smoke sensors. This would solve the problem of passengers being locked in the car and unable to escape due to fires caused by traffic accidents or vehicle electrical system failures [10]. Fuzzy logic control has now been widely applied in car design, including car suspensions [11,12], ABS brakes [13-15], hybrid electric vehicle engine power distribution [16,17], road deviation [18-20] and car following systems [21,22] etc.

The action of opening a car door involves multiple sensory perceptions, from using the eyes to judge whether there are vehicles approaching from the rear, to opening the car door handle when it has been confirmed that it is safe to do so, to pushing open the car door and getting out of the car, to closing the car door. This involves a series of coordinations of the muscular system, which are in response to perceptual stimuli. This is often known as procedural memory [23]. Martínez-García et al. proposed the idea of a feedback response of visual memory in tracking control [24]. In addition, visual perception errors, for instance, using the rear-view mirror to observe an oncoming vehicle and overlooking the speed of the vehicle, will also cause door crash risks due to a response delay [25]. This body of research will use sensors to track oncoming vehicles to help drivers avoid the danger of a door crash risk caused by careless procedural memory actions or visual perception errors that cause delays in action responses.

The purpose of this study is to develop and design a door lock control system under the circumstances of door crash prevention. When the driver stops and shifts the gear lever to the P position, before opening the door and getting out, the car sensor will detect the distance and speed of the motorcycle coming from the rear and determine whether the driver and passengers can open the door or use mandatory Dutch Reach to open the door via fuzzy logic control to ensure the safety of the driver and the motorcyclist alike. The rest of this article has been structured as follows: Section 2 describes the techniques and methods used. Section 3 explains the Vehicle Door Opening Model (VDOM). Section 4 explains the execution of the VDOM and discusses this. Finally, Section 5 summarises the main conclusions drawn.

\section{Technology and Method}

\subsection{Distance and Speed Detection}

The distance and speed detection technology in the design of this research can be directly referred to and adopts the blind spot detection system currently installed on the car. The major technology of the current blind spot detection system is roughly divided into two types: image and radar. The image-based blind spot detection system uses cameras mounted under the side and rear-view mirrors or at the rear of the car to detect cars and motorcycles on both sides and at the rear of the vehicle in an image-based manner [26]. Zhao et al. proposed a camera-based deep learning method that can accurately detect other vehicles in the blind spot. It transfers the blind spot detection calculation to the neural image classification work, which can boost the accuracy of sensing the approaching vehicles from the rear [27]. The disadvantage of the image-based blind spot detection technology lies in the weather. Weather conditions such as direct sunlight, rain, dense fog, spray and so on will cause the camera to fail. Additionally, night vision technology poses another problem. Failure of detection can also be caused by the lights of cars or motorcycles not being turned on at night [28]. The radar-based blind spot detection system, on the other hand, uses the microwave radar spectrum to install the radar sensor on the side or the rear of the car, and emit microwaves to detect incoming cars from the side and the rear. In comparison with imaging technology, microwaves do not rely on air transmission, hence, the detection capability of microwaves is relatively unaffected by climate. Rodenbeck and 
Beun proposed that a 94-GHz microwave radar is used to detect small-scale vibrations behind parked vehicles, which can distinguish whether the engine of the rear vehicle is running, and whether the rear vehicle is in a stationary or driving mode. It can also detect whether the door of the parked vehicle is opened in order to alert the driver [29]. Currently, commercially available blind spot detection systems (BSD) rely on two types of short-wave radar spectrums: $24 \mathrm{GHz}$ and $77 \mathrm{GHz}$. The $24 \mathrm{GHz}$ detection angle is 60 degrees, the detection distance ranges from 0.2 metres to 50 metres, and the $77 \mathrm{GHz}$ has a detection angle of 30 degrees and a detection range of 10 metres to 250 metres [30]. Both of the above types of short-wave radars can be used in this research for the fuzzy logic detection control system to avoid door crashes.

\subsection{Fuzzy Logic}

Fuzzy set is also known as an uncertain set. In a typical set, things that can be clearly distinguished can be represented by 0 and 1, for example, male and female. However, it is difficult to make a crisp distinction between most things and semantic expressions, that is, vague and unclear narratives, and fuzzy sets are similar human thinking patterns, extending binary logic to multi-valued logic [31]. The membership of the fuzzy set is defined as a level between 0 and 1 . The membership function determines the level defined as follows:

$$
h_{A}(x): X \rightarrow\{0,1\},
$$

where $A$ is the fuzzy set defined in the universal set $X$. For instance, the concept of "high speed" or "low speed" can be represented by a fuzzy set. Its membership function defines the perception of high or low according to the value of speed [32]. Similarly, an approximate integer constitutes a normal and convex fuzzy set [33].

For the operation of fuzzy sets, in the universe of discourse, $A$ and $B$ are defined as fuzzy sets [34] as follows:

$$
\begin{aligned}
& \text { complement: } h_{\bar{A}}(x)=1-h_{A}(x) \\
& \text { intersection : } h_{A \cap B}(x)=h_{A}(x) \wedge h_{B}(x) \equiv \min \left[h_{A}(x), h_{B}(x)\right] \\
& \text { union: } h_{A \cup B}(x)=h_{A}(x) \vee h_{B}(x) \equiv \max \left[h_{A}(x), h_{B}(x)\right]
\end{aligned}
$$

Fuzzy inference is based on fuzzy logic, and the inference program includes fuzzy input and fuzzy relationship [35] as follows:

$$
\begin{aligned}
& \text { input: } x \text { is somewhat } A\left(x=A^{\prime}\right) \\
& \text { rule: if } x \text { is } A \text { then } y \text { is } B \\
& \text { conclusion: } y \text { is somewhat } B\left(y=B^{\prime}\right)
\end{aligned}
$$

Among them, $A, A^{\prime}, B$, and $B^{\prime}$ are all or part of fuzzy sets, and the rule represents the fuzzy causal relationship between $x$ and $y$. The statement " $x$ is $A$ " found in the if part of the rule is referred to as a premise and the statement "then" is a consequence. The validity of the consequence is determined by the compatibility between an input and a premise. In other words, the true degree of " $\mathrm{y}$ is $\mathrm{B}$ " is determined by the degree of the compatibility between " $x$ is somewhat $A$ " and " $x$ is $A$ " [36].

A fuzzy inference system can be composed of more than one rule, and each rule is composed of more than one promise variable, as follows:

$$
\begin{aligned}
& \text { input: } x_{1}=A^{\prime} \text { and } x_{2}=B^{\prime} \\
& \text { rule } 1 \text { if } x_{1}=A_{1} \text { and } x_{2}=B_{1} \text {, then } y=C_{1} \\
& \text { rule } 2 \text { if } x_{1}=A_{2} \text { and } x_{2}=B_{2} \text {, then } y=C_{2} \\
& \vdots \\
& \text { rule } i \text { if } x_{1}=A_{i} \text { and } x_{2}=B_{i} \text {, then } y=C_{i} \\
& \text { conclusion: } y=C^{\prime}
\end{aligned}
$$


The compatibility check between the input and the premise $W_{i}$ of rule $i$ is as follows:

$$
W_{i}=\left\{\vee_{x_{1}}\left[h_{A}\left(x_{1}\right) \wedge h_{A_{i}}\left(x_{1}\right)\right]\right\} \wedge\left\{\vee_{x_{2}}\left[h_{B}\left(x_{2}\right) \wedge h_{B_{i}}\left(x_{2}\right)\right]\right\}
$$

In Equation (4), $\wedge$ indicates the minimum and $\wedge$ the maximum of the operands of universe of discourse. That is, the fuzzy set of $A$ and $B$ in this case. When a given input triggers $\mathrm{n}$ different rules, it calculates the compatibility between the input and the premise for each rule, then the conclusion is the average of each consequence. $C^{\prime}$ remains a fuzzy number [37], which requires defuzzification. Defuzzification is the process of obtaining a single value from the fuzzy set of $C^{\prime}$ and converting the result of fuzzy inference into crisp output. In other words, defuzzification relies on the decision-making calculations of the fuzzy set to calculate the best clarity value. Defuzzification is the action of converting $C^{\prime}(y)$, $y \in Y$ to a crisp value $\mathrm{y}^{*}$, that is, finding a clear point $\mathrm{y}^{*} \in Y$ that best represents the fuzzy set $C^{\prime}(y)$ for input. This research uses the Centre of Gravity Defuzzification Technique, which is the most commonly used method as well as more suitable for this research. It seeks the centre of gravity of the shadow area of the inference result, as follows:

$$
\mathrm{y}^{*}=\sum W_{i} \times C_{i} / \sum W_{i}
$$

\subsection{Mandatory Dutch Reach Method for Vehicle Door Opening}

The Dutch Reach method has been suggested as a potential solution to ensure that the vehicle driver is able to turn their body around to check if there are oncoming vehicles from behind. It is recommended that drivers use their "far hand", that is the hand furthest away from the door, to operate the door handle. The expectation is that this will force a natural change in the driver's posture, especially the orientation of their head. As a result, they are better able to see the sides and the rear of the vehicle, making it easier to spot potential danger nearby. Studies have found that using the Dutch Reach method to open the door will increase head rotation. It has also been found that drivers use the Dutch Reach method to open the door when they are specifically asked how they would open the door in a way that increases their awareness of any danger of an approaching vehicle from behind [38]. What has been termed the mandatory Dutch Reach vehicle door opening design is to add a "close hand" touch switch in the centre pillar of the car, near the seatbelt. Thus, the driver is forced to use their "far hand" to open the door. A conceptual diagram of the mandatory Dutch Reach car door opening method is shown in Figure 1.

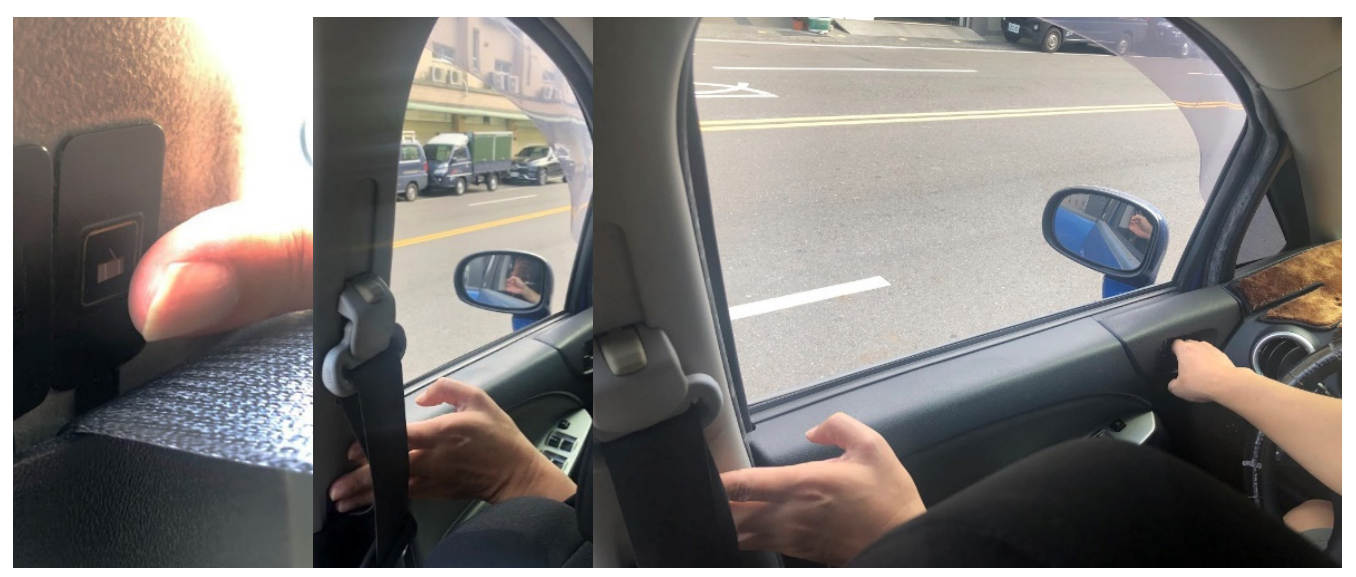

Figure 1. Schematic diagram showing the mandatory Dutch Reach vehicle door opening method.

\section{Vehicle Door Opening Model (VDOM)}

\subsection{Concept and Structure of the Fuzzy Logic System}

The core concept behind the fuzzy logic controller is a heuristic approach of writing rules for operating the controller, mainly in the format of "if A then B". The fuzzy logic 
system usually comprises two units, a knowledge base and a fuzzy inference unit (FIU). The knowledge base involves previously verified real environment information; while the fuzzy inference unit uses the information in the database composed of rules to determine the answer to the input question $[39,40]$. The fuzzy logic control system used in this research is shown in Figure 2.

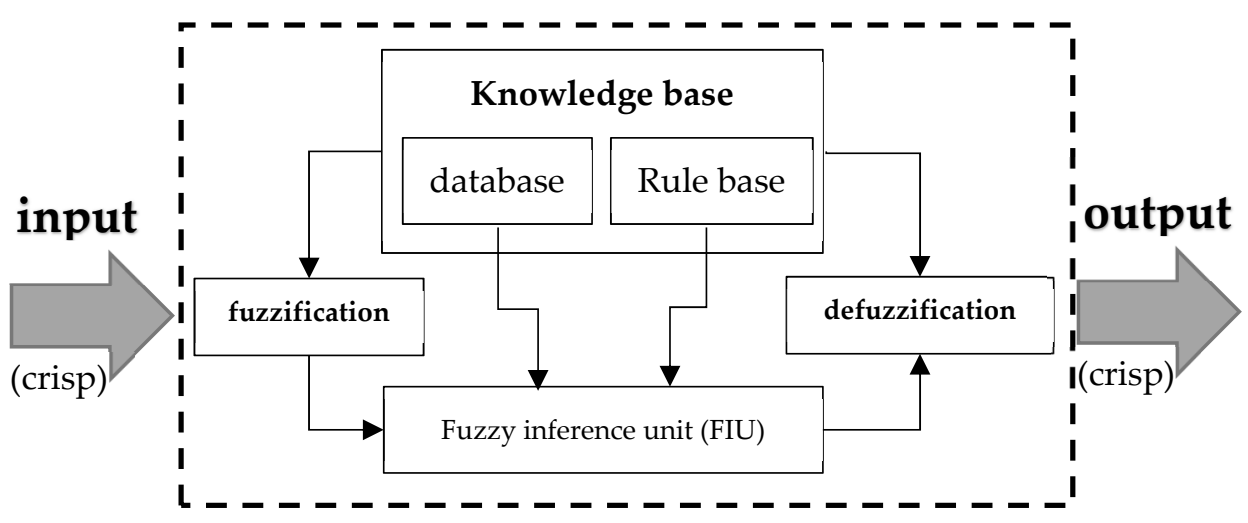

Figure 2. Fuzzy logic control system.

Fuzzification converts the input information into a fuzzy logic data format that can be expressed in words and crisp values into fuzzy values. The knowledge base accumulates the set of conclusion rules used to reach the decision. It covers a database and a rule base. The database is used to define the fuzzy membership function used in fuzzy rules; the rule base, on the other hand, contains fuzzy IF-THEN rules to practicalise the knowledge. Fuzzy inference systems produce fuzzy results using knowledge base rules based on input processed by fuzzification. The reasoning method adopted in this study is mamdani [41] Defuzzification converts the fuzzy value into a crisp value based on the fuzzy result produced by the fuzzy input of fuzzy decision making. This research adopts the Centre of Gravity Defuzzification Technique. Based on the requirements of the fuzzy logic controller design, the fuzzy rules are set to two input variables and one output variable, which includes the input of the motorcycle speed, the input of the safety distance, and the control system and level output. In order to prevent the car door being locked and unable to be opened if the sensor on the VDOM malfunctions, especially in a situation where emergency escape is required, the doors of the VDOM system must have an emergency escape door switch for the driver to use in the event of a VDOM failure and needing to escape the vehicle in an emergency. Figure 3 shows the framework of the VDOM, and Figure 4 shows a schematic diagram of the Emergency Vehicle Door Release Lever.

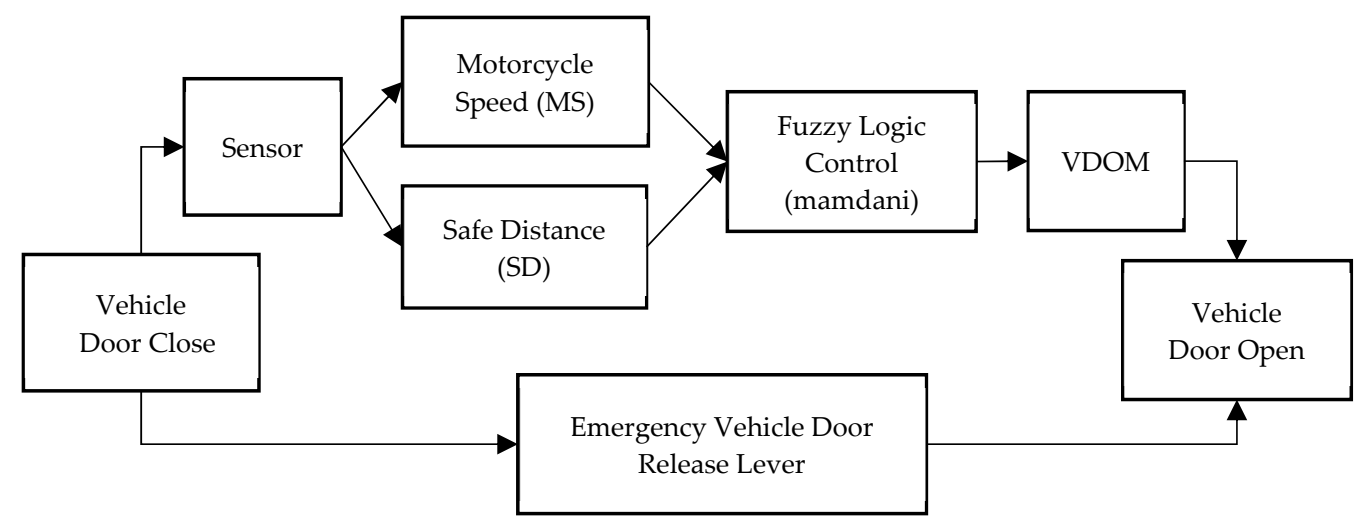

Figure 3. Structure of VDOM. 


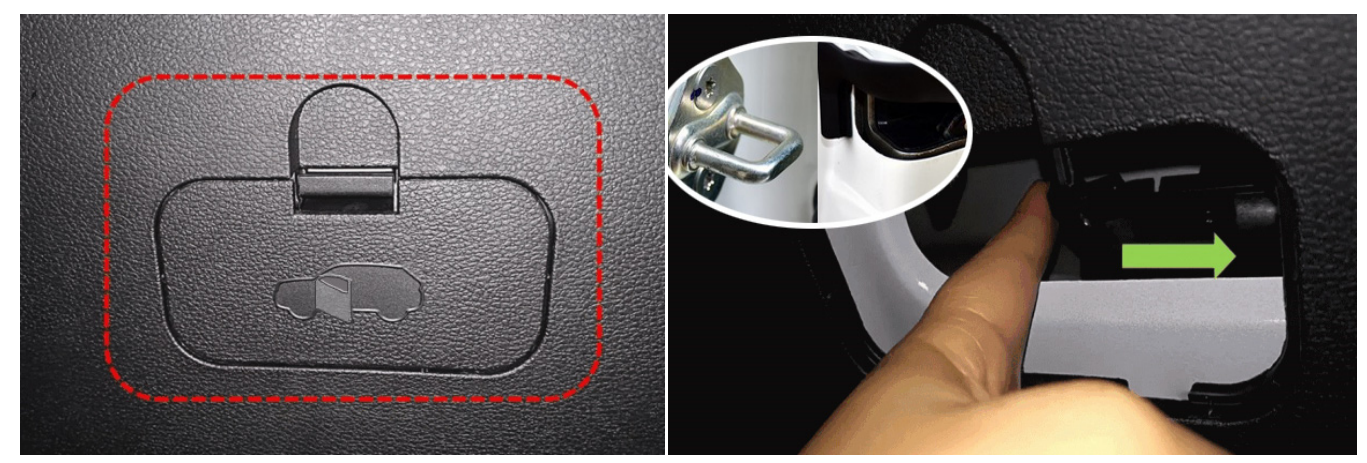

Figure 4. Schematic diagram showing emergency vehicle door release lever.

\subsection{Input and Output Variables}

Two input variables are defined in the control system design, namely, motorcycle speed (MS) and safety distance (SD). The input value is sent to the Fuzzy Inference Unit (FIU), then the most suitable result is found from the defined rule system, and transformed into a crisp output variable via defuzzification, which is the Vehicle Door Opening Model (VDOM). The door lock, switch and voice operating conditions are then controlled by the car's central control system. The fuzzy controller input fuzzy set, output fuzzy set, membership function and linguistic expressions are illustrated in Table 1.

Table 1. VDOM input and output membership functions.

\begin{tabular}{ccc}
\hline \multicolumn{2}{c}{ Input Variables } & Output Variables \\
\hline $\begin{array}{c}\text { Motorcycle Speed } \\
\text { (MS, km/h) }\end{array}$ & Safety Distance (SD, m) & $\begin{array}{c}\text { The Vehicle Door Opening } \\
\text { Model (VDOM, Level) }\end{array}$ \\
\hline Very slow (VS, 0-20) & Very close (VC, 0-9) & Very dangerous (VD, 0-1) \\
Slow (S, 10-30) & Close (C, 5-13) & Dangerous (D, 0-2) \\
Medium (M, 20-40) & Medium (M, 9-17) & Medium (M, 1-3) \\
Fast (F, 30-50) & Far away (A, 13-21) & Safe (S, 2-4) \\
Very fast (VF, 40-50) & Very far away (VA, 17-21) & Very safe (VS, 3-4) \\
\hline
\end{tabular}

\subsubsection{Motorcycle Speed Input}

The motorcycle speed input refers to the speed of the motorcycle approaching a parked vehicle from the rear. The basic strategy of the traffic safety system approach is to ensure that in the event of a collision, the impact energy is kept below a critical value that may cause death or serious injury. This threshold varies from one collision scenario to another, depending on the level of protection provided to the relevant road users. For instance, when a vehicle exceeds $30 \mathrm{~km} / \mathrm{h}$, the chance of survival for unprotected pedestrians in a collision drops drastically. As for motorcyclists, the threshold for collision speed is $50 \mathrm{~km} / \mathrm{h}$. According to research by the Organization for Economic Cooperation and Development (OECD), due to the difference in level of protection for pedestrians, cyclists, and motorcyclists, when the collision speed increases from $30 \mathrm{~km} / \mathrm{h}$ to $50 \mathrm{~km} / \mathrm{h}$, the fatality rate will see an exponential increase of between $10 \%$ to $80 \%$. As illustrated by the dotted line in Figure 5, motorcycles have a high degree of fragility, meaning the speed of the motorcycle is a key factor impacting its safety [42]. Figure 3 combines pedestrian and motorcyclist mortality rates [43] and a motorcycle speed membership function diagram. Referring to the documents mentioned above, the motorcycle speed membership function covers a range of $0-50 \mathrm{~km} / \mathrm{h}$ and is divided into five levels from very slow (VS) to very fast (VF). 


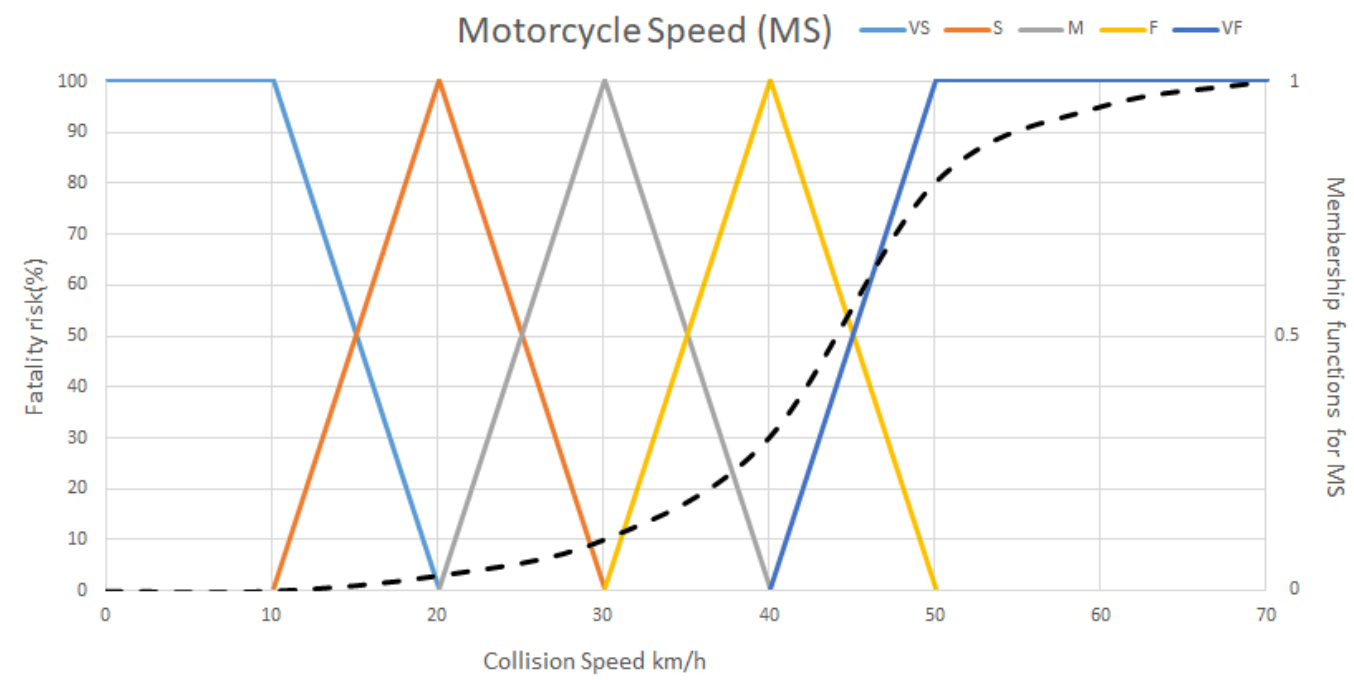

Figure 5. Death risk from collision speed and motorcycle speed membership function.

\subsubsection{Safe Distance Input}

The safe distance input refers to the distance of a motorcycle approaching a parked vehicle from the rear. Generally speaking, in Taiwan, attentive car drivers will usually use the rear-view mirror to observe whether there is an approaching motorcycle before opening the door and getting out of the car [44]. Therefore, car drivers possess a psychological threshold value for safe distance. This study has conducted an experiment on behavioural observation. In order to obtain the critical value of the driver's safe distance when opening the door, 51 test subjects were recruited, and each test subject was tested 3 times to measure their minimum safe distance from a vehicle coming from behind, that is, instead of opening the door, they will wait until the vehicle passes if said vehicle is within a safe distance. The behavioural observation experiment results found that the test subjects' psychological safe distance of opening the door was from 5-25 m, the average being $13 \mathrm{~m}$, the standard deviation was $4.4 \mathrm{~m}$, and the cumulative percentage is shown in the black dotted line in Figure 6. Figure 6 is a membership function diagram that combines the subjects' cumulative percentage of safe distance and the safe distance. The membership function of the safe distance covers a range of 0 to $25 \mathrm{~m}$. The safe distance medium function is set as the mean plus or minus the standard deviation, with a range of about 9 to $17 \mathrm{~m}$. The entire membership function is divided into five levels from very close (VC) to very far away (VA).

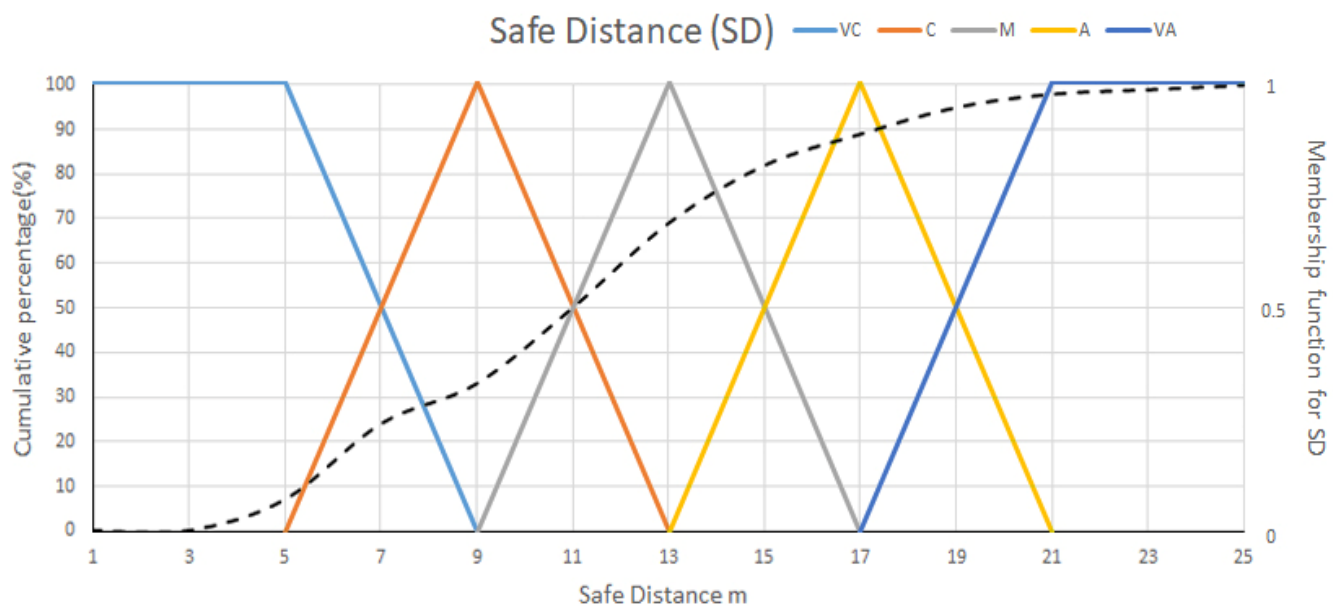

Figure 6. The cumulative percentage of safe distance and its membership function. 


\subsubsection{Vehicle Door Opening Model (VDOM) Output}

It is incredibly dangerous when a motorcycle is approaching at high speed and is close in distance to the car and the door is opened by the driver. This often results in the motorcyclist being seriously injured and even death upon second collision. The car driver is obliged to bear the corresponding responsibility. In situations where the motorcycle is approaching fast but the distance is relatively long, there may be a buffer space for the motorcyclist to manoeuvre. Therefore, the variables in the output are defined by the level of danger. There are five grades of membership functions ranging from very dangerous (VD) to very safe (VS). The membership function diagram of the fuzzy set output is shown in Figure 7.

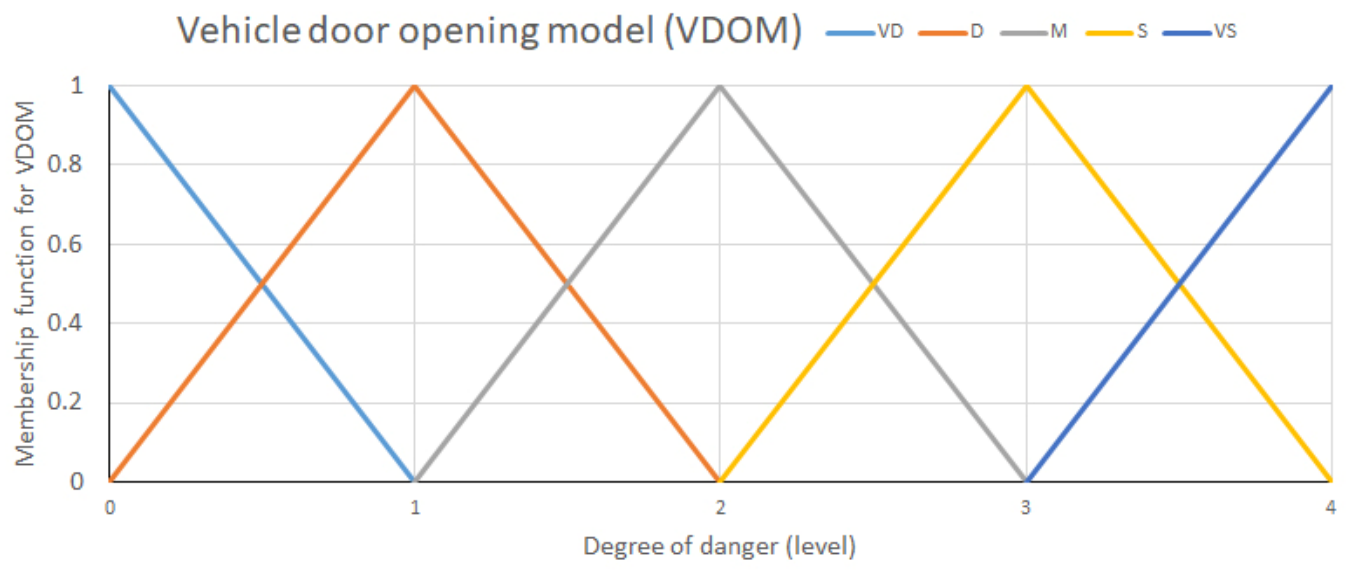

Figure 7. Membership function for VDOM.

\subsection{Rule Base and Defuzzification}

Each rule is a conditional sentence of "if $x_{1}=A_{1}$ and $x_{2}=B_{1}$, then $y=C_{1}$ ", which means that the conditions represented by the two sets of prerequisite variables are the input, and the output result is predictable. A fuzzy reasoning system makes decisions using the rules provided in the rule base. The rules used in this study were created through behavioural observations and the results of related research investigations. In this study, 25 rules were created using membership functions, such as Rule 1: if MS $=\mathrm{VF}$ and $\mathrm{SD}=\mathrm{VC}$ then $\mathrm{VDOM}=\mathrm{VD}$; Rule 13: if $\mathrm{MS}=\mathrm{M}$ and $\mathrm{SD}=\mathrm{M}$ then $\mathrm{VDOM}=\mathrm{M}$; and Rule 25: if $\mathrm{MS}=\mathrm{VS}$ and $\mathrm{SD}=\mathrm{VA}$ the VDOM $=\mathrm{VS}$. The rules table is shown in Table 2.

Table 2. VDOM input and output membership functions.

\begin{tabular}{|c|c|c|c|c|c|c|c|}
\hline \multirow{2}{*}{ if } & & \multicolumn{5}{|c|}{ Motorcycle Speed(MS) } & \multirow{2}{*}{ Then } \\
\hline & & VF & $\mathbf{F}$ & $\mathbf{M}$ & $\mathrm{S}$ & VS & \\
\hline \multirow{5}{*}{ Safe Distance(SD) } & VC & VD & VD & $\mathrm{D}$ & $\mathrm{D}$ & $\mathrm{M}$ & \multirow{5}{*}{ VDOM } \\
\hline & $\mathrm{C}$ & VD & $\mathrm{D}$ & $\mathrm{D}$ & M & $S$ & \\
\hline & M & $\mathrm{D}$ & $\mathrm{D}$ & M & S & $\mathrm{S}$ & \\
\hline & A & $\mathrm{D}$ & M & $S$ & $S$ & VS & \\
\hline & VA & M & $S$ & $S$ & VS & VS & \\
\hline
\end{tabular}

In this study, the Centre of Gravity Defuzzification Technique is adopted as it is the most commonly used method in related research. In this method, calculating the output of each membership function and the corresponding maximum membership value $\mathrm{y}^{*}$ is a clear and crisp number. The $y^{*}$ after defuzzification in this study will be between 0 and 4 . Today, when a driver shifts into P gear, the central control lock of a car automatically opens, and the driver can turn the door handle manually after turning the engine off to leave the car. In the VDOM mode, when the driver has parked the car and is ready to get out, the 
system for detecting incoming vehicles from the rear starts to work to determine whether there is an approaching motorcycle behind. After inputting the speed and distance of the motorcycle, through means of Fuzzy Inference Unit (FIU), crisp values are output and divided into three modes, namely, Danger, Caution and Warning. When $\mathrm{y}^{*}$ is a value between 0 and 2, the central lock enters Danger mode. At this time, the central lock is closed. The vehicle driver needs to use mandatory Dutch Reach to open the door and it will voice "danger"; when $\mathrm{y}^{*}$ is between 2 and 3 , the central control lock enters Caution mode. At this time, the central control lock is closed, one can manually unlock it and it will voice "caution"; when $\mathrm{y}^{*}$ is between 3 and 4 , the central control lock enters Warning mode, and at this time, the central control lock is open and one can manually open the door and it will voice "warning".

\section{Execution of the VDOM and Discussion}

When the car driver switches to the P gear and is about to get out of the car, the sensor for detecting the speed and distance of the motorcycle behind is activated. After entering Motorcycle Speed (MS) and Safe Distance (SD) and then using the IF-THEN rule base and the fuzzy inference unit (FIU), the output VDOM fuzzy set membership function is obtained. Figure 8 shows the response surface mesh of the input and output relationship of the fuzzy inference unit (FIU).

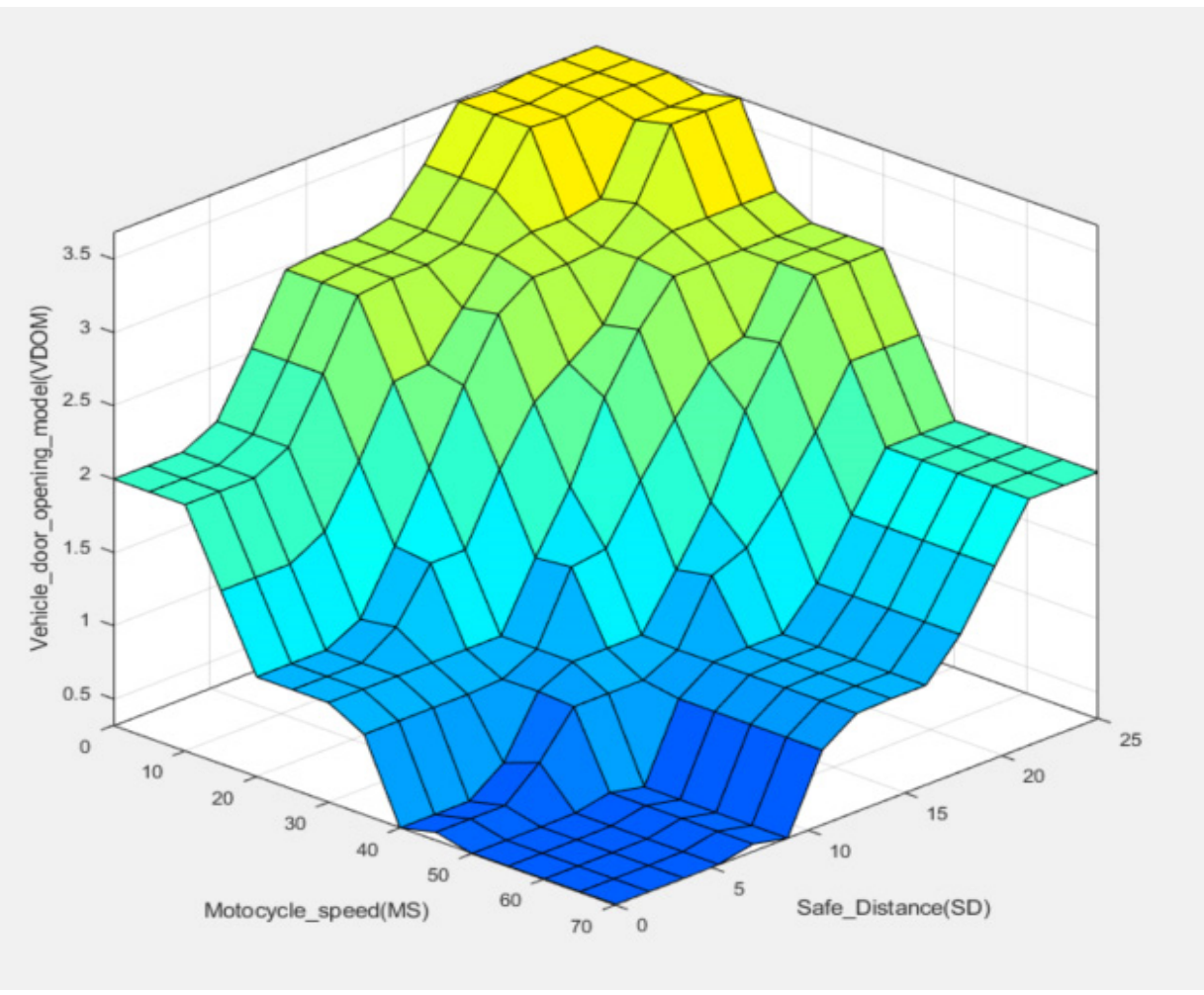

Figure 8. Response surface mesh of the VDOM.

Through the Centre of Gravity Defuzzification Technique, the value of the membership function of each rule presents the maximum membership degree and the weighted average, and a crisp defuzzification value can be obtained. The value is between 0 and 4 , and the results show the feedback response of the driver opening the door under different conditions. For instance, in Case 1, when the rear motorcycle is approaching at a speed of $50 \mathrm{~km} / \mathrm{h}$ (VF) and the safe distance is 25 metres (VA), the obtained VDOM value is 2, which belongs to Danger mode. The central control lock is closed, and the vehicle driver needs to use mandatory Dutch Reach to open the door and it will voice "danger", as shown in Figure 9. In Case 2, when the rear motorcycle approaches at a speed of $30 \mathrm{~km} / \mathrm{h}(\mathrm{M})$ 
and the safe distance is 25 meters (VA), the obtained VDOM value is 3, which belongs to Caution mode. At this time, the central control lock is closed, the lock can be opened manually, and it will voice "caution", as shown in Figure 10. As for Case 3, when the rear motorcycle is approaching at a speed of $30 \mathrm{~km} / \mathrm{h}(\mathrm{M})$ and the distance is 13 meters $(\mathrm{M})$, the obtained VDOM value is 2 . At this time, it belongs to Danger mode, and the central control lock is closed. The vehicle driver needs to use mandatory Dutch Reach to open the door and it will voice "danger", as shown in Figure 11. Given the circumstances of Case 1 and Case 3, if the driver rashly opens the door, the motorcyclist only has 1.5-1.8 s of reaction time, which is likely to cause a door crash accident. Motorcyclists in Case 2, however, will have a relatively sufficient reaction time of $3 \mathrm{~s}$, so the VDOM operation result is reasonable and appropriate. Figures 9-11 contain 25 rules from top to bottom. On the left side is the input of Motorcycle Speed, in the middle is the input of Safe Distance. The yellow block represents the membership function of the input value. The result obtained by FIU defuzzification falls on the right at the VDOM output result, which is presented by the blue block.

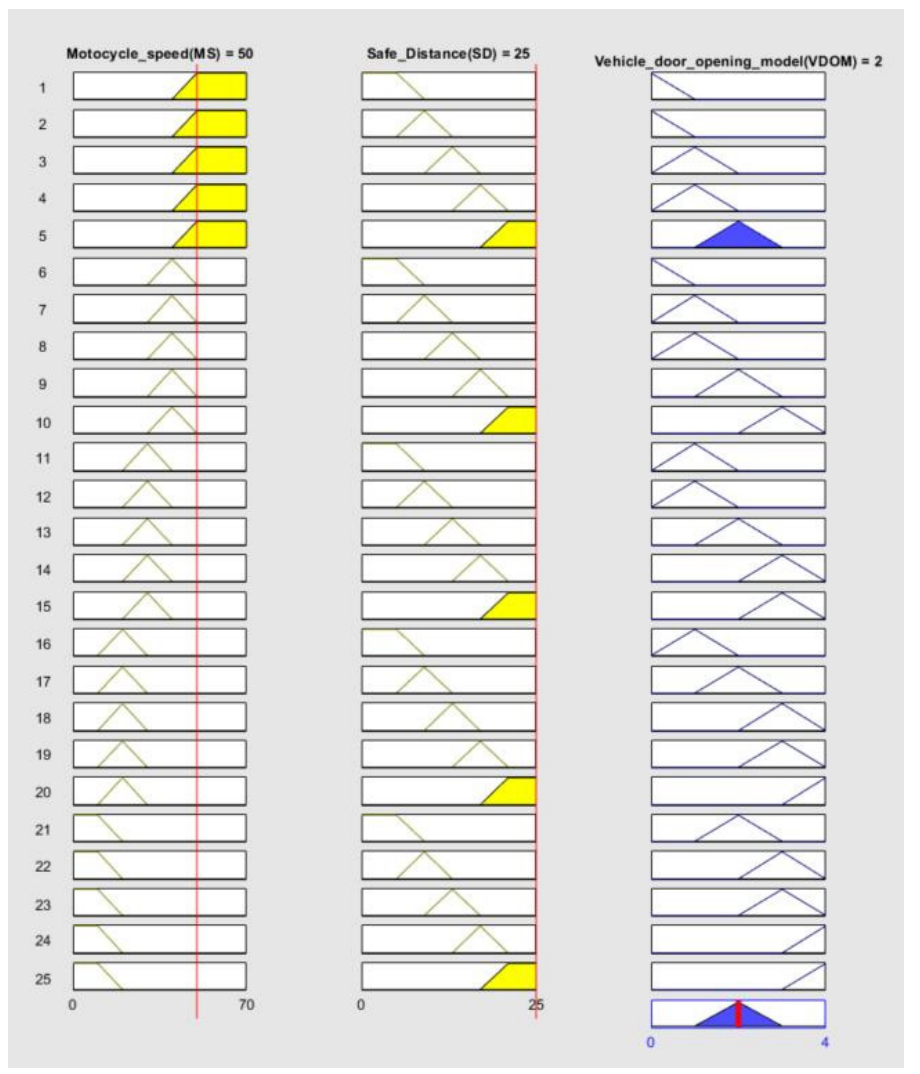

Figure 9. $\mathrm{MS}=50, \mathrm{SD}=25$, and $\mathrm{VDOM}=2$. 

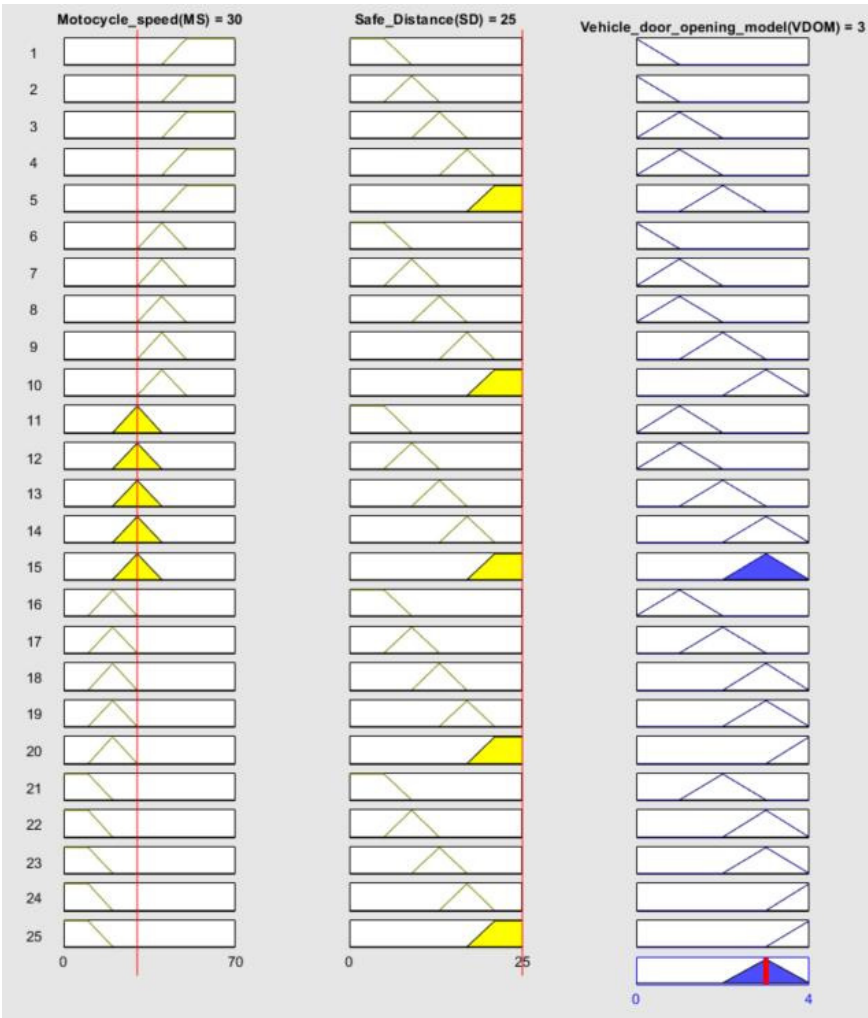

Figure 10. $\mathrm{MS}=30, \mathrm{SD}=25$, and $\mathrm{VDOM}=3$.

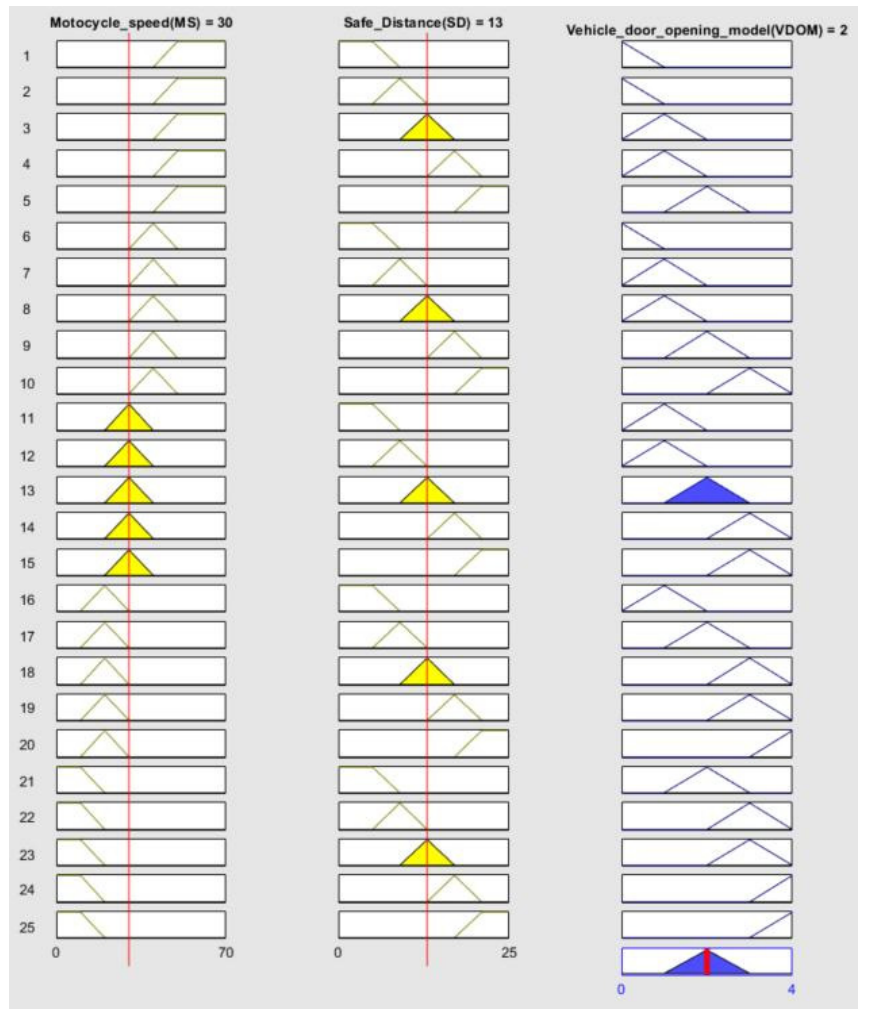

Figure 11. $\mathrm{MS}=30, \mathrm{SD}=13$, and $\mathrm{VDOM}=2$.

This research proposes the application of a fuzzy logic control system in car door controls to prevent door crash accidents. When a car is parked and is shifted to the P gear, 
the sensor is activated to detect if there is a motorcycle in close proximity coming from the rear. Motorcycle speed (MS, 0-70 km/h) and safe distance (SD, 0-25 m) are inputs, and additionally, the fuzzy inference unit (FIU) calculates the crisp fuzzy value of the vehicle door opening mode (VDOM, level 0-4) to control the opening of the door. When a fast-approaching motorcycle clocks in at a speed of $50 \mathrm{~km} / \mathrm{h}$ or above, the VDOM will fall into Danger mode, and the vehicle driver needs to use mandatory Dutch Reach to open the door. This is to ensure the driver turns his body to pay attention to approaching vehicles from behind to avoid door crash accidents. When the motorcycle is at a medium speed of $30 \mathrm{~km} / \mathrm{h}$ and the distance is far, it will first be in Caution mode, and the door requires manual unlocking. When the safety distance is less than $13 \mathrm{~m}$, it will enter Danger mode. When the speed of the motorcycle is very slow at $10 \mathrm{~km} / \mathrm{h}$ and the distance is far, it is in Warning mode. The door can be opened directly, but there will be a voice warning. When the safety distance is less than $13 \mathrm{~m}$, it will switch into Caution mode. When the safety distance is less than $5 \mathrm{~m}$, it will enter Danger mode. This way, a door crash accident can be effectively avoided. The VDOM level change diagram of motorcycle speed at different safety distances is shown in Figure 12. At present, this body of research proposes a simple method of detecting motorcycle speed and safe distance to either give a warning to the driver who wants to get out of the car or make it difficult for him to open the door. Future research will also consider more input factors for the VDOM fuzzy logic control to make a judgement with. This includes more accurate vehicle image recognition and a more precise image recognition range, to avoid detection errors and failures that cause the driver to be unable to open the door. Additionally, we can follow the movements, reactions, and eye tracking of the driver who wants to get out of the car. This is to ensure that the driver is paying attention to whether or not there is a vehicle approaching from the rear before he opens the door to get out of the car and is not looking at or talking on his phone and getting out of the car as a procedural memory action, leading to the risks resulting from a delayed action response caused by errors in visual perception. In the future, the author will construct a series of behaviour observation experiments for the VDOM. The author will create a VDOM system in a virtual scene and observe the test subjects' behavioural responses to the VDOM through VR as a verification experiment for the system.

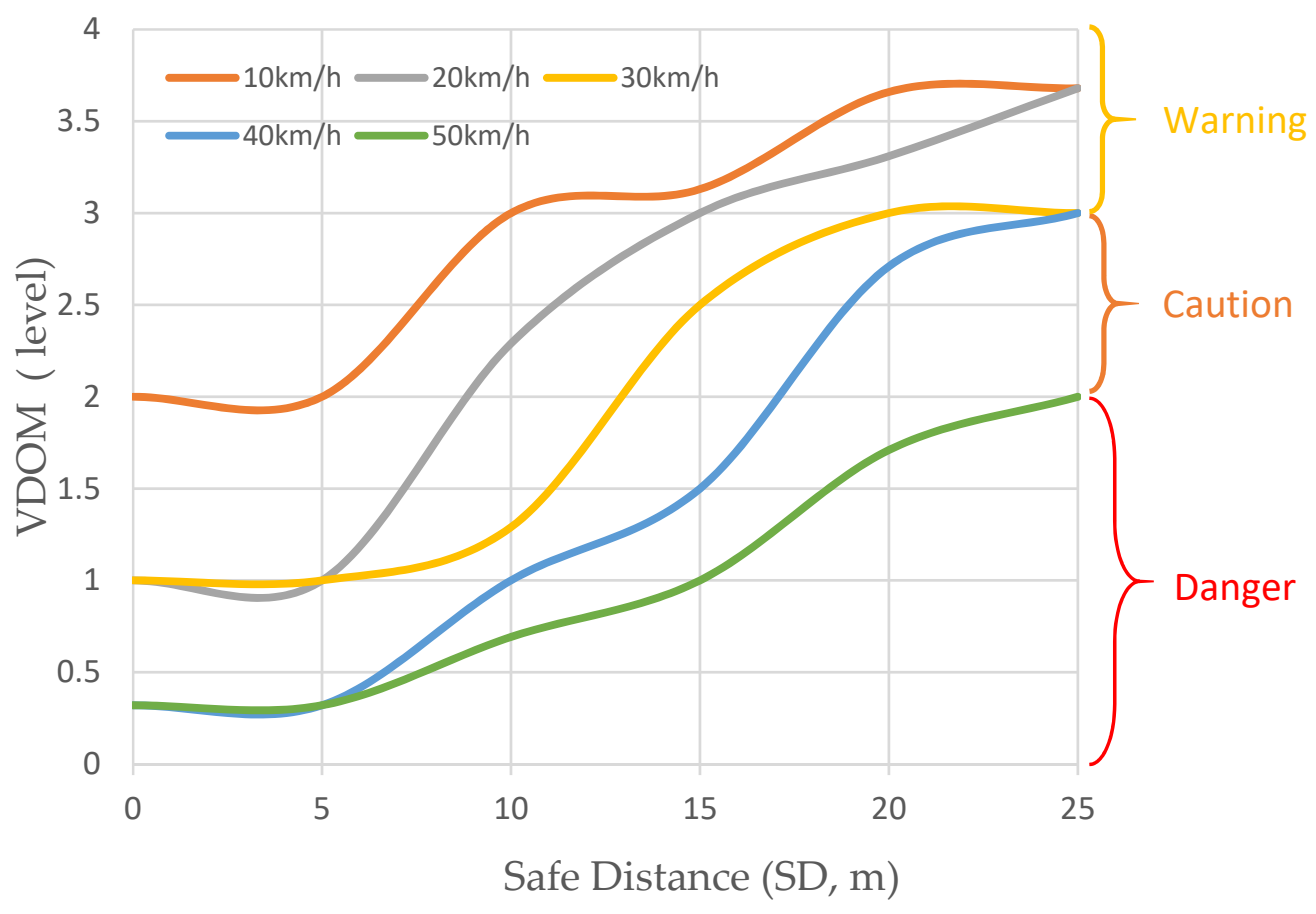

Figure 12. VDOM level change diagram when different motorcycle speeds are close to the parked vehicle. 


\section{Conclusion}

This research has suggested the use of a VDOM method to avoid motorcycle-vehicle door crashes and has studied fuzzy logic control. First, it has discussed the possible technologies which could be used, including image detection technology and microwave detection technology, which can meet the requirements of detecting the distance and speed of an approaching motorcycle from the rear when the vehicle driver opens their door. Then, fuzzy logic control has been used to control the related vehicle door opening mechanisms. Subsequently, the concept of fuzzy logic and its calculation method have been discussed, including how to find the crisp output value by inputting the data and rule base, then using the Centre of Gravity Defuzzification technique. The VDOM takes into account the distance and the speed of the approaching motorcycle from the rear, and a crisp value is obtained through entering the information and the rule base into the fuzzy inference unit to be defuzzified. There are a total of 25 rules in the rule base. The membership function of the speed of the motorcycle approaching from the rear has been formulated with reference to the OECD's fatality rates for motorcycle riders according to their speed. The safe distance has been determined through experimental data, which tested what vehicle drivers deemed to be the critical value of safe distance between the car door and something approaching from the rear. The implementation and discussion of the VDOM has been presented in Section 4 by presenting three different motorcycle speeds and safe distances to understand the results of the execution output of the VDOM. Through the VDOM level change diagram of different motorcycle speeds at different safe distances, the suitability of the design of the VDOM can be seen, showing that the VDOM can certainly be effective in avoiding motorcycle-vehicle door crash accidents.

Funding: This research received no external funding.

Institutional Review Board Statement: Not applicable.

Informed Consent Statement: Not applicable.

Data Availability Statement: Provided by the author upon request.

Conflicts of Interest: The author declare no conflict of interest.

\section{References}

1. Chen, P.-L.; Wang, M.-H.; Linkov, V.; Pai, C.-W. Motorcycle door crashes: An evaluation of crash characteristics in Taipei City, Taiwan. PLoS ONE 2018, 13, e0208016. [CrossRef] [PubMed]

2. Pai, C.-W.; Hsu, J.-J.; Chang, J.-L.; Kuo, M.-S. Motorcyclists violating hook-turn area at intersections in Taiwan: An observational study. Accid. Anal. Prev. 2013, 59, 1-8. [CrossRef] [PubMed]

3. Pai, C.-W. Overtaking, rear-end, and door crashes involving bicycles: An empirical investigation. Accid. Anal. Prev. 2011, 43, 1228-1235. [CrossRef] [PubMed]

4. Huang, C.-Y. An investigation of motorist behavior in Taiwan. Adv. Hum. Asp. Transp. Part I 2014, 7, 303.

5. Kalra, N.; Anderson, J.; Wachs, M. Technical Report Documentation Page; PATH Research Report: Berkeley, CA, USA, 2009.

6. Kusano, K.D.; Gabler, H.C. Potential Occupant Injury Reduction in Pre-Crash System Equipped Vehicles in the Striking Vehicle of Rear-end Crashes; Association for the Advancement of Automotive Medicine: Chicago, IL, USA, 2010; Volume 54, pp. $203-214$.

7. Yadav, A.; Shah, S.R. Role of Human Factors in the Design of Blind Spot Detection Technology. Int. Res. J. Eng. Technol. 2020, 7, 1026-1030.

8. Wankhade, P.P.; Dahad, S.O. Real time vehicle locking and tracking system using GSM and GPS technology-an anti-theft system. Int. J. Technol. Eng. Syst. (IJTES) 2011, 2, 272-275.

9. Siyal, K.; Gugapriya, G. Anti-theft vehicle locking system using CAN. Indian J. Sci. Technol. 2016, 9, 1-6. [CrossRef]

10. Mathavan, J.J.; Faslan, A.W.; Basith, N.U.A.; Wanigasinghe, W.V.S.D. Hardware Implementation of Fire Detection, Control and Automatic Door Unlocking System for Automobiles. In Proceedings of the 2020 4th International Conference on Trends in Electronics and Informatics (ICOEI) (48184), Cheranmadevi, India, 15-17 June 2020; pp. 125-129.

11. Sakman, L.E.; Guclu, R.; Yagiz, N. Fuzzy logic control of vehicle suspensions with dry friction nonlinearity. Sadhana 2005, 30, 649-659. [CrossRef]

12. Guclu, R. Fuzzy logic control of seat vibrations of a non-linear full vehicle model. Nonlinear Dyn. 2005, 40, 21-34. [CrossRef]

13. Lee, Y.; Zak, S.H. Designing a genetic neural fuzzy antilock-brake-system controller. IEEE Trans. Evol. Comput. 2002, 6, 198-211.

14. Kokes, G.; Singh, T. Adaptive fuzzy logic control of an anti-lock braking system. In Proceedings of the 1999 IEEE International Conference on Control Applications (Cat. No.99CH36328), Kohala Coast, HI, USA, 22-27 August 1999; Volume 1, pp. 646-651. 
15. Pramudijanto, J.; Ashfahani, A.; Lukito, R. Designing neuro-fuzzy controller for electromagnetic anti-lock braking system (ABS) on electric vehicle. J. Phys. Conf. Ser. 2018, 974, 012055. [CrossRef]

16. Derakhshan, M.; Shirazi, K.H. Optimized fuzzy controller for a power-torque distribution in a hybrid vehicle with a parallel configuration. Proc. Inst. Mech. Eng. Part D J. Automob. Eng. 2014, 228, 1654-1674. [CrossRef]

17. Won, J.S.; Langari, R. Fuzzy torque distribution control for a parallel hybrid vehicle. Expert Syst. 2002, 19, 4-10. [CrossRef]

18. Boada, B.L.; Boada, M.J.L.; Diaz, V. Fuzzy-logic applied to yaw moment control for vehicle stability. Veh. Syst. Dyn. 2005, 43, 753-770. [CrossRef]

19. Ahmed, A.A.; Jomah, O.S.M. Vehicle Yaw Rate Control for Lane Change Maneuver Using Fuzzy PID Controller and Neural Network Controller. In Proceedings of the 2020 IEEE 2nd International Conference on Electronics, Control, Optimization and Computer Science (ICECOCS), Kenitra, Morocco, 2-3 December 2020; pp. 1-6.

20. Pan, S.; Zhou, H. An adaptive fuzzy PID control strategy for vehicle yaw stability. In Proceedings of the 2017 IEEE 2nd Information Technology, Networking, Electronic and Automation Control Conference (ITNEC), Chengdu, China, 15-17 December 2017; pp. 642-646.

21. Ghaffari, A.; Khodayari, A.; Kamali, A.; Tajdari, F.; Hosseinkhani, N. New fuzzy solution for determining anticipation and evaluation behavior during car-following maneuvers. Proc. Inst. Mech. Eng. Part D J. Automob. Eng. 2018, 232, 936-945. [CrossRef]

22. Bennajeh, A.; Bechikh, S.; Said, L.B.; Aknine, S. A fuzzy logic-based anticipation car-following model. In Transactions on Computational Collective Intelligence XXX; Springer: Berlin/Heidelberg, Germany, 2018; pp. 200-222.

23. Squire, L.R. Memory systems of the brain: A brief history and current perspective. Neurobiol. Learn. Mem. 2004, 82, 171-177. [CrossRef]

24. Martínez-García, M.; Zhang, Y.; Gordon, T. Memory Pattern Identification for Feedback Tracking Control in Human-Machine Systems. Hum. Factors 2019, 63, 210-226. [CrossRef] [PubMed]

25. Zhang, Y.; Martinez-Garcia, M.; Gordon, T. Human Response Delay Estimation and Monitoring Using Gamma Distribution Analysis. In Proceedings of the 2018 IEEE International Conference on Systems, Man, and Cybernetics (SMC), Miyazaki, Japan, 7-10 October 2018; pp. 807-812.

26. Sotelo, M.Á.; Barriga, J. Blind spot detection using vision for automotive applications. J. Zhejiang Univ.-Sci. A 2008, 9, 1369-1372. [CrossRef]

27. Zhao, Y.; Bai, L.; Lyu, Y.; Huang, X. Camera-Based Blind Spot Detection with a General Purpose Lightweight Neural Network. Electronics 2019, 8, 233. [CrossRef]

28. Fernández, C.; Llorca, D.F.; Sotelo, M.A.; Daza, I.G.; Hellín, A.M.; Álvarez, S. Real-time vision-based blind spot warning system: Experiments with motorcycles in daytime/nighttime conditions. Int. J. Automot. Technol. 2013, 14, 113-122. [CrossRef]

29. Rodenbeck, C.T.; Beun, J.B. Identifying the operation of a parked car's engine, transmission, and door using millimetre wave pulse Doppler radar. Electron. Lett. 2020, 56, 959-961. [CrossRef]

30. Ramasubramanian, K.; Ramaiah, K. Moving from Legacy $24 \mathrm{GHz}$ to State-of-the-Art 77-GHz Radar. ATZelektronik Worldw. 2018, 13, 46-49. [CrossRef]

31. Zadeh, L.A. Fuzzy sets as a basis for a theory of possibility. Fuzzy Sets Syst. 1978, 1, 3-28. [CrossRef]

32. Kikuchi, S.; Chakroborty, P. Car-following model based on fuzzy inference system. Transp. Res. Rec. 1992, 1365, 82-91.

33. Dubois, D.J. Fuzzy Sets and Systems: Theory and Applications; Academic Press: Cambridge, MA, USA, 1980 ; Volume 144.

34. Zadeh, L.A. Fuzzy sets. In Fuzzy Sets, Fuzzy Logic, and Fuzzy Systems: Selected Papers by Lotfi A Zadeh; World Scientific: Singapore, 1996; pp. 394-432.

35. Kaufmann, A.; Gupta, M.M. Introduction to Fuzzy Arithmetic: Theory and Applications; Van Nostrand Reinhold Company: New York, NY, USA, 1991.

36. Oussalah, M. On the compatibility between defuzzification and fuzzy arithmetic operations. Fuzzy Sets Syst. 2002, 128, 247-260. [CrossRef]

37. Dubois, D.J.; Prade, H.; Yager, R.R. Readings in Fuzzy Sets for Intelligent Systems; Elsevier Science: Amsterdam, The Netherlands, 2014.

38. Large, D.; Choo, M.C.; Houghton, R. Validating 'Dutch Reach': A Preliminary Evaluation of Far-Hand Door Opening and its Impact on Car Drivers' Head Movements. In Proceedings of the 7th International Cycling Safety Conference, Barcelona, Spain, 10-12 October 2018; pp. 1-11.

39. Jang, J.-S. ANFIS Adaptive-Network-based Fuzzy Inference System. IEEE Trans. Syst. Man Cybern. 1993, 23, 665-685. [CrossRef]

40. Kunhimangalam, R.; Ovallath, S.; Joseph, P.K. A Novel Fuzzy Expert System for the Identification of Severity of Carpal Tunnel Syndrome. BioMed Res. Int. 2013, 2013, 846780. [CrossRef]

41. Mamdani, E.H.; Assilian, S. An experiment in linguistic synthesis with a fuzzy logic controller. Int. J. Man-Mach. Stud. 1975, 7, 1-13. [CrossRef]

42. OECD/ITF. Towards Zero: Ambitious Road Safety Targets and the Safe System Approach; Organisation for Economic Co-Operation and Development: Paris, France, 2008.

43. Wramborg, P. A New Approach to a Safe and Sustainable Road Structure and Street Design for Urban Areas. In Proceedings of the 13th International Conference on Road Safety on Four Continents, Warsaw, Poland, 5-7 October 2005; Volume 13, p. 12.

44. Huang, C.-Y. Observations of Drivers' Behavior when Opening Car Doors. Procedia Manuf. 2015, 3, 2753-2760. [CrossRef] 